\title{
Research on Quality Index System of Digital Aerial Photography Results
}

\author{
Wencong Jiang ${ }^{1}$, Yanling $\mathrm{Li}^{1}$, Yong Liang ${ }^{1}$, and Yanwei Zeng ${ }^{2}$ \\ ${ }^{1}$ School of Information Science \& Engineering, Shandong Agricultural University, Taian, \\ Shandong 271018, P. R. China \\ ${ }^{2}$ State Supervision and Testing Center of Surveying and Mapping Product, \\ 1982nd Section Renmin Road, Chengdu 610081, China \\ liyanling@sdau.edu.cn
}

\begin{abstract}
Aerial photogrammetry is one of the main methods obtaining geospatial information. After entering the 21st century, aerial photogrammetry technology has fully entered the digital age, and the quality of digital aerial photography results will directly affect the quality and accuracy of results of surveying and mapping. Therefore, it is necessary to inspect the quality of digital aerial photography results. However, in the practical application, the production and quality inspection of digital aerial images have not had a complete norm to guide all the time. So in the subsequent application, image data appears lots of problems. Based on development situation at home and abroad and national existing regulation, generalize and establish a complete quality index system has a far-reaching significance in perfecting digitalization and informatization surveying and mapping technological system. The contents of quality inspection of aerial photography results are comprehensive, which include data quality, flying quality, the image quality and annex quality. From the perspective of productive practice, combined with practical experience of scanning digitalization production and a series of experiences of the quality inspection process of digital aerial images, the article focuses on the process to establish a complete quality index system.
\end{abstract}

Keywords: digital photogrammetric image, aerial photography results, film scanning, quality inspection, quality index.

\section{Introduction}

With the rapid development of surveying and mapping and the advancement of computer technology and CCD technology, aviation digital cameras have become the main technical means of a high-resolution and high-precision aerial remote sensing and uncontrolled aerial survey. Digital aerial photography has shown obvious advantages, especially in large area, large-scale and speedy mapping, and digital aerial image map, which has some features of rich information and intuitive truth, is widely used in surveying and mapping fields. With more and more mature digital aerial photography technology and more and more results, the quality of aerial photographs directly 
determines the mapping precision, aesthetics, reliability and current situation. In the process of establishing informatization surveying and mapping system, as the important data source of the basic geographic information data, digital aerial photography results will become more and more, and "real-time and automation of data processing" of informatization surveying and mapping requires automation and rapid response of quality inspection and evaluation, which proposes a challenge how to check the quality of digital aerial photography results. At present, quality inspection index of the aerial photography results distributes in the relevant national and industry standards and quality inspection index of the quality inspection and evaluation of digital aerial photography results does not form a complete system; still using manual methods inspects the quality of digital aerial photography results, so the above technical means are backward. However, digital aerial photography provides image data which makes it possible to use computer programs making more comprehensive and rigorous automatic quality inspection. At present, scanning the original negatives to acquire digital images is still the primary means of digital image acquisition. The digital system of aerial imaging aerial photo scanner scans film to acquire digital aerial image map. Therefore, quality inspection of aerial images inherits the traditional inspection content and increases some contents of the aspects of image digitalization ${ }^{[1]}$.On the basis of collecting research results of the quality inspection of digital aerial photography results at home and abroad, understanding new developments of flight quality inspection and image quality inspection of digital aerial photography results, and analyzing the current situation of quality inspection technology of aerial photography results at home and abroad, according to national relevant norms, standards and regulations etc, it is essential to research and establish a perfect quality inspection index system of digital aerial photography results.

\section{Quality Inspection Index of Digital Aerial Photography Results}

The contents of quality inspection of digital aerial photography results are comprehensive, which include data quality flying quality, the image quality and annex quality. According to the four aspects, and analysis of quality inspection elements of digital aerial photography results, it is reasonable and scientific to establish a complete quality inspection index system of digital aerial photography results.

\subsection{Data Quality}

Data quality includes: aerial camera testing data, negative pressing detection information, and aerial designing data.

\subsubsection{Arial Camera Testing Data}

Generally, we can use wide-angle aerial camera with $152 \mathrm{~mm}$ focal length. In order to meet the need for elevation measurement precision or have some difficulties of height of airplane flight in high altitude, we can use special wide-angle aerial camera with $88 \mathrm{~mm}$ focal length. 
We should use the same main distance aerial camera in aerial photography zonings of the same area. For large areas of the community, we can use up to aerial cameras of three different main distance, but we must use the same main distance aerial camera in the same route.

Under the cloud photography we should use aerial camera of ensuring high-quality.

Aerial camera should be check by infection department with appropriate qualification. Items and accuracy of infection are in the Table 1.

Table 1. The accuracy test

\begin{tabular}{cc}
\hline items & accuracy \\
\hline checking the main distance & $\pm 0.005 \mathrm{~mm}$ \\
radial distortion & $\pm 0.005 \mathrm{~mm}$ \\
coordinates of optical mark & $\pm 0.003 \mathrm{~mm}$ \\
coordinates of best symmetric main point & $\pm 0.005 \mathrm{~mm}$ \\
the main point of auto-collimation & $\pm 0.005 \mathrm{~mm}$ \\
\hline comprehensive resolution of the lens & no less than 25 line pair per millimeter \\
\hline shutter speed & $1 / 100 \mathrm{~s} \sim 1 / 1000 \mathrm{~s}$ \\
\hline
\end{tabular}

\subsubsection{Negative Pressing Detection Information}

The quality of aerial photography data affects mapping precision. In the process of aerial photography, we do not only to strictly carry out the aerial plan and aerial design, but also flying qualities and photographic quality is crucial. Inspection of Negative pressing accuracy is indispensable.

Each cassette of photo zone should be checked two or four continuous flat area photographs. Selecting good quality of negative images, the normal overlapping, small obliquity and rotation angle, and clear and complete frame mark images are important. Distance of orientation point (standard point) to the azimuth line should be not less than $9.5 \mathrm{~cm}$, and the checkpoint should be evenly distributed.

Analytical method is used to inspect the quality of the negative pressing, according to the principles of photogrammetry, after two or four consecutive stereo image pairs, which are needed to check, make azimuth line orientation with the precise three-dimensional coordinate measuring apparatus, exterminating the orientation point and check point's coordinates and parallax of each image pairs, and then the computer can be used to make relative orientation calculation of consecutive stereo image pairs. While calculating the relative orientation elements, it must check the size of the remaining parallax from top to bottom of the model orientation points and checkpoints to determine the negative pressing situations (generally not more than $0.02 \mathrm{~mm}$, the individual points more than $0.03 \mathrm{~mm})^{[2]}$. 


\subsubsection{Aerial Design Data}

In aerial photography design work, aerial photography can be deal with automation by computer, and can obviously enhance the reliability of data and improve data accuracy to ensure quality control of the design work effective. The superiority can not be compared with calculation by hand.

\subsection{Image Quality}

The overall requirements of image quality are as follows. Good image must have some features, such as moderate contrast, color saturation, clear frame mark, histogram with normal distribution, the image histogram values in the 0-255 gray scale and consistent color in adjoining flight course. Image quality includes the quality of image resolution, color mode, image clarity and frame mark, image color, geometric accuracy and integrity of the image, richness of veins information, data formats and compression standards.

\subsubsection{Image Resolution}

Image resolution is the main parameter of reflecting the amount of information of digital image. Currently, acquisition of digital aerial images is basically through the use of high precision aerial photo scanner, so we use the scanning resolution to measure the size of image resolution, which is different from ground resolution. Scanning resolution is that a pixel on digital images corresponds to the size of the original film. In general, the scanning resolution of image should ensure the scale of 15-25um. Of course, the determination of image resolution is also based on photography scale of aerial photograph, mapping scale and the quality of the original aerial photograph. Typically, the image resolution of a project should be consistent, otherwise in the subsequent photogrammetric operations, the software system will not normally display threedimensional model and can not match images.

\subsubsection{Color Mode}

Color model is the type of showing image color, that is the binary mode, grayscale mode, and color mode etc. color models can also be expressed as RGB color space and CMYK color space and so on.

\subsubsection{Image Clarity and Frame Mark}

Image clarity expresses the clarity of the edge of images. Image clarity is the main indicators of reflecting image quality. Clear images are not only to help interpret surface features, but also to improve capacity of visual identification and the accuracy and to improve the success rate of image matching. Images are not clear mainly due to the following aspects: Unlearned images may be the cause of the original photograph or pressed glass not pressing film in the process of scanning; Image pixel is rough, and generally decomposition of the original photograph is too low, and silver halide particles are too large, or sampling parameter is not set to the best in the process of scanning; Impurities on the photograph may be due to improper handling with the fingerprints, 
hair, scratches and other marks in the process of photography, flushing, preservation and scanning.

Images and the frame mark are clear directly determine eligibility of the scanning data. Usually, checking image clarity and frame mark is through the means of visual view and contrasting. Frame mark basically is divided into two categories, such as the "crosshairs" and "center point". Checking the frame mark is at least to enlarge $100 \%$ of canning pixel with the digital image to check. If the outline of cross wire or center point is clear, the scanning is qualified, otherwise it is needed to see the frame mark of original film or check the scanner flatten device. Inspection of clarity of the image requires a combination with film image quality checklist to check. Usually, the clarity of image is closely related to the clarity of original photograph. If the film is clear, scanning image should be clear, however, the clarity of scanning image is affected by the image gray level and contrast, and gray level and the contrast which are too large or too small will affect the visual perception judgment of image clarity. In such special circumstances, it should be carefully examined, combined with histogram of gray level and other inspection tools, to see whether to meet the requirement after treatment.

\subsubsection{Image Color}

The color requirements of digital aerial images are as follows. Good image must have some features. Brightness and contrast are well-distributed and moderate, and image histogram which is normal distribution is full of 256 gray scales as much as possible. Image brightness and contrast which are too big or too small will result in the loss of information. Color aerial images must be paid special attention to color balance, especially for identification and inspection of the color of key surface features on the photograph the pivotal features on. For example: on the true color aerial photograph, green vegetation displays as green, while on the color infrared aerial photo, it is dark red.

Image color should be paid special attention to the color difference of within and between photographs. The image tone and color of all photograph of the surveyed area are consistent, and internal color of sole photograph should be consistent, especially not appearing the apparent color seams in the photograph because of the scanner failures.

\subsubsection{Geometric Accuracy and Integrity of the Image}

Image geometric accuracy mainly depends on the size of the errors which are caused by photography and scanning. Photographic apparatus and scanner which are used for aerial photographs must be a dedicated instrument. Especially the scanner must be image digitizing system which is the high precision and high resolution. A variety of geometric information on the original film should reflect on digital images. The geometric precision of the scanner image can make calculation of interior orientation on the photograph, and usually the interior orientation residuals of photographs can not more than the size of half-pixel.

Image integrity mainly ensures the integrity of photograph, and the integrity of the marginal additional information and frame box. The frame box of aerial photograph is an important symbol to determine the coordinates of digital images and the reference point position of the frame box and the additional information of the photographical 
edge should be complete and clear, in order to easily understand the parameters of the camera lens.

\subsubsection{Richness of Veins Information}

Veins information of digital aerial image is a comprehensive reflection of spatial characteristics of objects, and integrated veins information is foundation of obtaining special subject information extraction. Digital aerial images should have rich veins information, fine veins information, and clear veins information.

\subsubsection{Data Formats and Compression Standards}

TIF format is international universal image format, and the general image scanners and processing software can be supported. But the different versions of TIF data may affect the software processing capability of data. At present, TIFF6.0 format is universal, and it can support a variety of compression modes. Of course, some software manufacturers have developed their own data formats and compression methods which are used in the specific software, so some users need this particular data format.

Data storage check: the file name should be normally consistent with the scanned image, that is, scanning data file name were named according to the number of film. Scanning data file formats are usually uncompressed * tif format. Black and white data is 256 gray scales and color data is RGB24bit. Checking stored data is correct, whether according to requirement scanning data is stored in the corresponding carrier or not, and the number of stored data files should be consistent with the source file number.

\subsection{Flight Quality}

Flight quality includes the quality of longitudinal overlap and sidelap, the absolute loopholes, relative loopholes, and supplemental photography of loopholes, angle of inclination and rotation angle of photo, flight course curvature, the keep of flight height, photo area, subarea map profile coverage guarantee, and control of routes.

\subsubsection{Longitudinal Overlap and Sidelap}

In relatively flat terrain areas, longitudinal overlap of photographs usually should be $60 \%-65 \%$, and individually the maximum is not more than $75 \%$ and the minimum is not less than $56 \%$; sidelap usually should be $30 \%-35 \%$. When elevation of adjoining flight course is large and can not satisfy the sidelap requirements, subarea aerial photography are required. The deviation of flight route and designing route can not more than $10 \%$ of the width of photograph cover; the difference of actual photographic scale and design scale is not more than $5 \%$. Because of the effects of air onflow, instability of airplane flight will cause that the main axis of the camera deviates from the vertical line, and the general deviation is not more than $3^{\circ}$.

\subsubsection{The Absolute Loopholes, Relative Loopholes, and Supplemental Photography of Loopholes}

When the value of longitudinal overlap and sidelap is 0 , it can be called the absolute loopholes. When the value of longitudinal overlap and sidelap is less than specified 
requirements, it can be called the relative loopholes. In aerial photography, the value of the photograph overlap is small or the photograph has no overlap part, it can be called the aerial photography loopholes. Aerial photography data does not allow having any loopholes. Absolute loopholes and relative loopholes are both needed to make supplemental photography in time, and supplemental photography must according to original design requirements to do, and the length of supplemental photography route should be beyond a baseline of outside of the loopholes length ${ }^{[3]}$.

\subsubsection{Angle of Inclination and Rotation Angle of Photo}

In the process of flight, changes of the adjoining photograph $<$ rotation angle $>$ generally are not more than 12 degrees. Angle of inclination of photo is controlled by the level control knob of the camera, and generally is not more than 3 degrees. During aerial photography, the bias correction and aircraft inclination will generate overrun of angle of inclination and rotation angle.

\subsubsection{Flight Course Curvature}

Flight course curvature is the greatest degree of deviation of ligature, which connects main points of photograph within a photographic flight course with main points of the first and last photograph. Flight course curvature is not more than $3^{\circ}$.

\subsubsection{The Keep of Flight Height}

The difference of the actual flight height and design flight height within subarea is not more than $5 \%$ of design flight height. In the same flight route, the difference of the maximum flight height and the minimum flight height is usually not more than $50 \mathrm{~m}$. when aerial photograph scale is more than or equal to 1:8000, in the same flight route the difference of the maximum flight height and the minimum flight height is not more than $30 \mathrm{~m}$, and flight height difference of the adjoining photo is not more than $20 \mathrm{~m}^{[4]}$.

\subsubsection{Photo Area, Subarea Map Profile Coverage Guarantee}

Splicing photograph by overlap arrangement, it is essential to check coverage situation in photo area which is marked on topographic maps.

Photo area boundary coverage guarantee: Coverage of flight routes which is beyond photo area boundary lines is not less than one baseline. Coverage of direction of side which is beyond photo area boundary lines is not less than 50\% of frame of image, and not less than $30 \%$ of frame of image at least. When routes are laid according to the center lines of maps and public map profile lines of two adjacent maps of direction of side, direction of side is beyond photo area boundaries which is not less than $12 \%$ of frame of image at least.

Subarea coverage guarantee: If direction of routes in adjoining subarea is the same, direction of side is the normal flight and direction of course is beyond a baseline of subarea boundaries. If direction of routes in adjoining subarea is not the same, direction of course is beyond a baseline of subarea boundaries and direction of side is beyond subarea boundaries which is not less than $30 \%$ of frame of image, and not less than $15 \%$ of frame of image at least; When routes are laid according to the center lines of maps 
and public map profile lines of two adjacent maps of direction of side, direction of side is beyond subarea boundaries which is not less than $12 \%$ of frame of image at least.

Map profile coverage guarantee: As the photo area boundary lines and subarea boundary generally coincide with the map profile lines, requirements of the map profile coverage are as above.

\subsubsection{Control of Routes}

In the photographic survey area, to reduce the layout of the photograph control point, control of routes is many adding flying routes which are approximate vertical with mapping routes. It is essential to check photograph scale of control routes, overlap, coverage, the absolute loopholes, relative loopholes.

\subsection{Annex Quality}

It is important to check the correction, integrity and complete, and standard of the documentation. data includes technical design, design summary, inspection reports, photographs index map, aerial identification table, number, annotation, packaging, data transfer books (aerial design books, photo area books, photos combination chart and so on), the measurement of photo behavior feature of aerial film and aerial photographic film washing records, scanning data, inventory of scanning data, design of scanning production, scanning table and records of problems and so on ${ }^{[5]}$.

\section{Results and Discussion}

\subsection{Results of Forming a Complete Quality Index System}

According to the above discussion and analysis of the quality elements and their specific parameters of digital aerial photography results, a more complete quality index system of digital aerial photography results can be created.

\subsection{Discussions}

In the process of production of aerial image, lots of factors affect the quality of aerial image, such as film density and the quality of washing and the scanning of aerial photograph and determination of resolution.

\subsubsection{Film Density and the Quality of Washing}

In the process of aerial camera work, it is through the air suction device to ensure the film leveling, at the time of the instantaneous exposure, and the difference is \pm 0.0005 " on the focal flat. In the film's preservation period, it should be a storage environment which is secure for sensitive material. In the preservation, transportation and the process of use, it should be paid attention to moistureproof, and it can not be exposed to places which are directly shinned by sunlight or have high temperature. 
Table 2. Elements of quality of digital aerial photography results

\begin{tabular}{cc}
\hline the first quality element & the Secondary quality element \\
\hline data quality & aerial camera testing data, negative pressing \\
image quality & image resolution, color mode, image clarity and \\
frame mark, image color, geometric accuracy and \\
integrity of the image, richness of veins information, \\
data formats and compression standards \\
longitudinal overlap and sidelap, the absolute \\
loopholes, relative loopholes, and supplemental \\
photography of loopholes, angle of inclination and \\
rotation angle of photo, flight course curvature, the \\
keep of flight height, photo area, subarea map \\
profile coverage guarantee, control of routes \\
the accuracy, completeness and standardization of \\
the document
\end{tabular}

The imaging and photographic fixing of photograph should use treatment control conditions recommended by manufacturer, and residual sulfur compounds, which have been handled, are not more than $0.04 \mathrm{mg}$ per square inch according to international common standards. Processed film should not be defective, torn and worn, and should not have watermarks, fingerprints, hair, dirt and other circumstances affecting image quality ${ }^{[6]}$.

\subsubsection{The Scanning of Aerial Photograph and Determination of Resolution}

Acquisition of digital images is that scanner makes the process of digitalizing film images. High-precision digital imaging system is used to scan aerial image, and inherent geometric accuracy of the scanner is at least $7 \mathrm{um}$, and the maximum scanning resolution should reach $15 \mathrm{um}$. Digital imaging system is commonly used at home, such as VX4000, 5000 model, the DPW scanning system of Leica / Helava, Imatizer of China Surveying and Mapping Institute, GeoSystem system of Ukraine, Photoscan of I / Z Corporation and so on. The main parameters of the process of scanning image for conclude file name, data format, compression type and compression ratio, scanning resolution, the color type, file size, image pyramid, the file header and color tone, etc, and tone color is the focus of quality control . many scanners are through setting up light transmission rate of a transparency film and Camma parameters to adjust brightness and contrast of the output digital imaging.Therefore, in the process of work, according to colors of photograph, we can make the definition of the maximum light transmission rate, minimum light transmission rate and Camma parameters in order to make output image color tone even and moderate.

The accuracy of operation should be paid attention in the process of scanning. For example, Film must be pressed flatten, and fingers do not directly touch the film, and it needs to keep temperature and humidity of the scanning room, and to maintain a clean 
working environment, etc. Moreover, it is important to avoid appearing fuzzy, hair, dirt, fingerprints of the output image and other phenomena. CCD width of many scanners is $5 \mathrm{~cm}$ below, so it should need multi-band scanning for back and forth, which can cover an aerial photo. If CCD and scanner have the dust pollution, it will inevitably lead to color difference between images within the photograph ${ }^{[7]}$.

Apart from having the relationship with film itself, resolution of digital images has the relationship with the size of scanning pixel. The smaller the size of scanning pixel is, the higher the resolution of digital image. At the same time, unit images have the greater amount of data. Scanning resolution directly affects the accuracy of follow-up results.

\section{Conclusions}

In the twenty-first century, aerial photogrammetry technology has fully changed from the simulation method, analytical method to digital photogrammetry technology, and original data is digital aerial images instead of simulated photograph, and means and methods have been greatly improved. With more and more mature digital aerial photography technology and more and more results, as the basis data of aerial photogrammetry technology, the quality of aerial photographs directly determines the mapping precision, aesthetics, reliability and current situation. On the basis of collecting research results of the quality inspection of digital aerial photography results at home and abroad, understanding new developments of flight quality inspection and image quality inspection of digital aerial photography results, and analyzing the current situation of quality inspection technology of aerial photography results at home and abroad, according to national relevant norms, standards and regulations etc, it is essential to research and establish a perfect quality inspection index system of digital aerial photography results. The contents of quality inspection of digital aerial photography results are comprehensive, which include data quality flying quality, the image quality and annex quality. According to contents of the four aspects and analysis of quality inspection elements of digital aerial photography results, it is reasonable and scientific to establish a complete quality inspection index system of digital aerial photography results. Moreover, in the process of production of aerial image, lots of factors affect the quality of aerial image, such as film density and the quality of washing and the scanning of aerial photograph and determination of resolution. From the perspective of productive practice, combined with practical experience of scanning digitalization production and a series of experiences of the quality inspection process of digital aerial images, the article focuses on the process to establish a complete quality index system.

\section{References}

1. Yuan, G., Gu, B., Huang, J.: The quality control and inspection of digital aerial images. In: 2003 Academic Annual Meeting Album of Institute of Surveying and Mapping of Jiangsu Province (2003)

2. The implementation details of inspection and acceptance and quality assessment of national aerial photography products. National Mapping Bureau (2001) 
3. Zhang, Z., Zhang, J.: Digital Photogrammetry. Science and technology University of Surveying and Mapping of Wuhan press, Wuhan (1995)

4. Qiao, R., Sun, H., Li, X.: Photography and aerial photography. Wuhan University Press, Wuhan (2008)

5. Supplementary technical requirements of national aerial photography. National Mapping Bureau (May 2003)

6. Yu, G., Li, W., Lin, Z.: The Discussion of influence of quality of aerial film pressure level on the mapping accuracy. Surveying and Mapping and Spatial Geographical Information (April 2009)

7. Ren, J.: The quality control of scanning digital production of aerial film. In: The Research of Materials of Photographis Institute of China of and Application Academic Symposium 